\title{
Potential Use of Rosemary (Rosmarinus officinalis L.) Essential Oil as Anti-Bacterial and Anti -Algal
}

\author{
Ali M. Najem ${ }^{1}$ and Ibrahim J. Abed ${ }^{1 *}$ \\ ${ }^{I}$ Department of Biology, College of science, University of Baghdad, Baghdad-Al-Jadiria, Iraq.
}

\begin{abstract}
The activity of Rosmarinus officinalis (Rosemary) essential oil screened for multi medicinal or pharmaceutical and industrial purposes as an alternative for chemical solutions with long-term and devastating side effects. Eicherichia.coli, Pseudomonas aeruginosa, Klebsiella pneumoniae, Staphylococcus aureus causes severe infections because it's difficult to be treated and lead to persistent of infection, these pathogens have been developed a defense mechanism against a broad spectrum of antibiotics threatens the global public health. The highest antibacterial activity of the Rosmarinus officinalis against E.coli $\left(M I C=10^{4} \mu \mathrm{g} / \mathrm{ml}\right.$ and $M B C=10^{4} \mu \mathrm{g}$ $(\mathrm{ml})$. Despite the splendid role of Chlorella sp. In production of biofuel basing on their high lipid containing, though, Chlorella and other algae have been became an obstacle to researchers in order to obtain unialgal culture (culture with only one species of algae) and our labs. were suffering from this problem during isolation of algae, 50,100,250,500 ppm from Rosemary essential oil were prepared. Results revealed very strong antagonistic effects of the essential oil concentrations on Chlorella vulgaris growth rates by measuring the amount of chlorophyll a. Generally growth rates decreased as the time and the concentrations of the essential oil increased comparing with the growth rate of standards (algae free from the essential oil) which exponentially increased along with time. Chlorella was sensitive to the allelopathic effect of this oil.
\end{abstract}

Keywords: anti-algal, anti -bacterial, Chlorella vulgaris, chlorophyll a, microdilution broth method, Rosmarinus officinalis

\section{Introduction}

Medicinal plants were used for centuries as remedies for human diseases because they contain components of therapeutic value, there is an increasing interest in phytochemicals as new sources of natural antioxidant and antimicrobial agents [1]. Phytochemicals are naturally occurring compounds of plant kingdom, such as medicinal plants, vegetables, fruits, that work with nutrients and fibbers to act against diseases or more specifically, provides protection against diseases [2]. Microbial infections have become one of the major problems of public health in the world and the development of resistance to the available antibiotics has lead researchers to investigate the antimicrobial activity of medicinal plants [3]. Essential oils have gained enormous interest as sources of natural products. They have been screened for their potential uses as alternative remedies for the treatment of many infectious diseases [4]. Rosmarinus officinalis L. belongs to the Lamiaceae family. This plant contains volatile oil, which has many potentialities in traditional medicine, cosmetics and flavoring agent in foods. Rosemary essential oil antangonistic activity as antibacterial effect for both Gram negative and positive bacteria, it's also antifungal, antioxidant, antimutagenic and shows cytotoxic activity[5]. Rosemary essential oil also possesses analgesic, anti-inflammatory, antioxidative, anti-tumor, anti-ulcerogenic and hepatoprotective properties [6] and as anti cyanobacterial [7]. Chlorella sorokiniana are adapted to grow in open systems and commercial production facilities are available. However, they are, less nutritive and contain hard cell wall [8]. Therefore, Chlorella vulgaris more accommodate to various environmental factors and that is precisely had been created an obstacle during unialgal culture, for this reason, eliminate Chlorella vulgaris by using phytochemical procedure is one of this research aims to purify the algal cultures.

\subsection{Extraction of volatile oil}

\section{Material and Methods}

Rosmarinus officionalis was collected from nursery nearby my Baghdad University campus; the plant was classified by college of science herbarium, then dried and finely ground. The volatile oil were extracted by hydro distillation with $300 \mathrm{~g}$ dry plant powder in $1.5 \mathrm{~L}$ for $5 \mathrm{~h}$., using Clevenger apparatus, the extracted oil was kept at $4^{\circ} \mathrm{C}$ until use [9].

\subsection{Culture of algae}

Chlorella vulgaris isolates were obtained from Ministry of Science and Technology laboratories and checked microscopically periodically in order to guarantee purification of the unialgal culture, an inoculums was cultured in CHU-10 media up to the exponential growth phase (7 days following inoculation) to obtain a high biomass prior the experiment [10]. 


\subsection{Evaluation anti- algal activity of Rosemary essential oil}

The algicidal activity of $R$. officionalis volatile oil was tested at four concentrations $(50,100,250,500$ ppm) monitored throughout 12 days against Chlorella vulgaris. The volatile oil was dissolved in DMSO (Dimethyl sulfoxide) and added to a flask containing $1 \mathrm{ml}$ of alga and $9 \mathrm{ml}$ of culture media (CHU-10). The culture condition (salinity, temperature, and light) during the experiments were the same. The density of alga was monitored every 2 days by estimating chlorophyll a (indirect method of Chlorella vulgaris biomass determination), the pigment can be completely extractable in solvents such as acetone and exhibits characteristic absorbance at $663 \mathrm{~nm}$, the estimation of chlorophyll a was estimated as follows: The Chlorella vulgaris culture centrifuged at 5000rpm for 10min. the pellet was re-suspended in $4 \mathrm{ml}$ of $80 \%$ acetone and mixed thoroughly. Tubes were incubated in a water bath at $60^{\circ} \mathrm{C}$ for $1 \mathrm{hr}$. in dark with occasional shaking, then the suspension was centrifuged at 5000rpm for $10 \mathrm{~min}$ and the supernatant was stored. Absorbance of the supernatant and $80 \%$ acetone as blank read at $663 \mathrm{~nm}$ in U.V. spectrophotometer. The amount of chlorophyll a in the sample was calculated using the formula 1 [11].

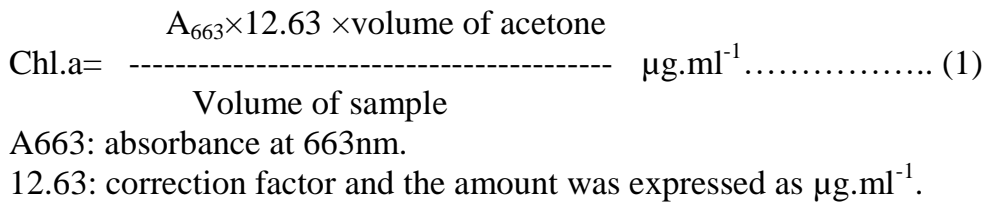

\subsection{Determination of minimal inhibitory concentration (MIC)}

E.coli, $P$. aeruginosa, $K$. pneumoniae, $S$. aureus isolates were taken from bacteriology laboratory in university of Baghdad-collage of science -Biology Department. The MIC values of Rosemary essential oil were evaluated using microdilution method in 96 well microtiter plate [12]. Two fold serial dilutions were prepared with brain heart infusion broth (Himedia/ India) in a final concentration ranging from $2 \times 10^{4}-256 \mu \mathrm{g} / \mathrm{ml}$ and the bacterial inoculums were added at final concentration $1 \times 10^{6}$ cell $/ \mathrm{ml}$. EO of Rosemary was dissolved in DMSO $\left(2 \times 10^{4} \mu \mathrm{g} / \mathrm{ml}\right)$ The results were recorded after incubation at $37^{\circ} \mathrm{C}$ for $18 \mathrm{~h}$ and measuring at $630 \mathrm{~nm}$ with an ELISA reader (BioTek). MIC (lowest concentration of the EO that inhibits the growth of bacteria) was determined by taking absorbance of the tested wells that did not exceed the value of the absorbance media control [13].

\subsection{Anti algal activity of Rosemary essential oil}

\section{Results and discussion}

Results recorded decline in the growth rate of Chlorella vulgaris, due to the allelochemical effect of Rosmarinus officinalis essential oil on these microalgae, represented by decreasing in the amount of chlorophyll a of Chlorella and that appeared in all tested concentrations $(50,100,250,500 \mathrm{ppm})$, growth rates of standards (algae free from the essential oil) representing by chlorophyll a concentration were exponentially increased along with time as shown in table (1). The algicidal effects of the rosemary essential oil increased as the time and concentrations of the essential oil increased except some irregular trends Chlorella showed a slightly resistance, generally uncountable with the remarkable drop in the amount of chlorophyll as shown in table (1).

Table-1- Variations in concentration of chlorophyll a of Chlorella vulgaris treated with Rosemary essential oil concentrations comparing with Chlorella free from oil (standard) along with time.

\begin{tabular}{|l|c|c|c|c|c|c|c|}
\hline Concentration of & \multicolumn{7}{|c|}{ Concentration of chlorophyll $(\mu \mathrm{g} / \mathrm{ml})$ along with time } \\
\cline { 2 - 9 } Rosemary oil ppm & 0.0 hr. (Initial) & $48 \mathrm{hr}$. & $96 \mathrm{hr}$. & $144 \mathrm{hr}$. & $192 \mathrm{hr}$. & $240 \mathrm{hr}$. & $288 \mathrm{hr}$. \\
\hline 0.0 (standard) & 0.18 & 0.27 & 0.222 & 0.359 & 0.343 & 0.105 & 0.0 \\
\hline 50 & 0.21 & 0.261 & 0.297 & 0.166 & 0.079 & 0.077 & 0.0 \\
\hline 100 & 0.199 & 0.291 & 0.497 & 0.255 & 0.1 & 0.167 & 0.0 \\
\hline 250 & 0.155 & 0.295 & 0.242 & 0.225 & 0.22 & 0.139 & 0.0 \\
\hline 500 & 0.22 & 0.281 & 0.299 & 0.171 & 0.116 & 0.047 & 0.0 \\
\hline
\end{tabular}

The essential oil highest inhibitory action was during (240 hr.) against Chlorella vulgaris according to the optical density readings (formulated into chlorophyll a concentration). The essential oil of Rosemary can be used as approach to purify algal cultures especially during isolating the cyanobacteria usually cyanobacteria need concentrations higher than Chlorella vulgaris to be inhibited, therefore, succession in using concentrations to inhibit Chlorella vulgaris lower than that against cyanobacteria in same mixture could be considered a step forward achieving an unialagal cultures of cyanobacteria. 500,1000 and1500 ppm of Rosemary essential oil revealed very strong antagonistic effects on M. aeruginosa and Chrooccocus minor growth rates by measuring the amount of chlorophyll- a of each alga [7]. Antagonestic activity of essential oils extracted from six 
submerged plants Potamogeton cristatus, P. maackianus, P. lucens, Vallisneria spiulosal, Ceratophyllum demersum and Hydrilla verticellata, showed high potency on M. aeruginosa [14].

\subsection{Antibacterial activity of Rosemary essential oil}

Antibacterial activity of rosemary essential oil clearly appeared in the light of the recorded results against $E$. coli, $P$. aeruginosa, and $K$. pneumoniae, $S$. aureus that was experimented by using MIC and MBC in a concentration $\mu \mathrm{g} / \mathrm{ml}$ as shown in Figure (1).

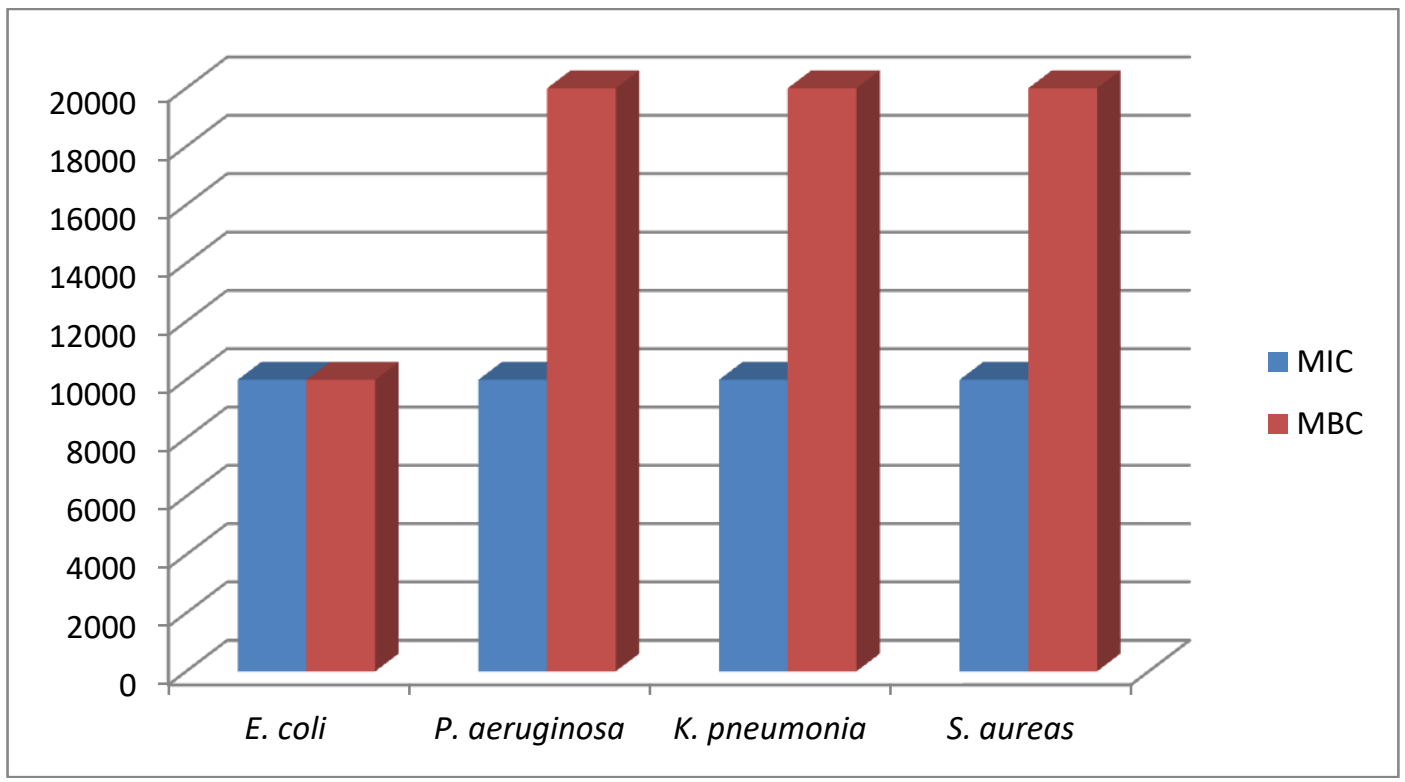

Figure (1) Antagonistic activity of Rosemary essential oil against E. coli, P. aeruginosa ,K. Pneumoniae, $S$. aureus by using MIC and MBC techniques in $\mu \mathrm{g} / \mathrm{ml}$.

Wang et al.[14] were investigated the antibacterial activity of rosemary essential oil on K. Pneumoniae in aiding of MIC technique, the results exhibited a remarkable impact on the biofilm of this bacteria by inhibition the initial cell attachment. Essential oil of rosemary showed antagonistic activity against 60 strains of multidrug resistant E.coli isolated from many specimens: the respiratory tract, abdominal cavity, urinary tract, skin and from hospital equipment. tested the antibacterial activity of 10 plant extracts included rosemary against 14 pathogenic microorganisms included E. coli, P. aeruginosa, K. Pneumoniae, S. aureus and MIC values for each were evaluated, the plant extract of rosemary showed no antagonistic activity against all the tested microorganisms except Bacillus subtilis and Candida albicans which were susceptible to the rosemary extract .[16]. According to [18] oils from Ocimum basilicum and Rosmarinus officinalis exhibited an antibacterial activity against the Gram-positive bacteria $S$. aureus, Enterococcus faecalisand, Listeria monocytogenes and against Gram-negative bacteria E. coli, Yersinia enterocolitica, Salmonella choleraesuis and P. aeruginosa as food borne bacterial strains. The rosemary essential oil strongly inhibits E. coli ATCC 25922 [18] and another study results showed that rosemary oil has an antibacterial effect on a number of microorganisms responsible for respiratory infections, isolated from clinical specimens, such as Streptococcus pyogenes, S. agalactiae, S. pneumoniae and K. pneumoniae, S. aureus and Stenotrophomonas maltophilia [20]. The essential oil from Rosmarinus tournefortii possesses antimicrobial activity against Gram-negative E. coli and P. aeruginosa and Gram-positive $S$. aureus [21]. A number of studies show that essential oils and their constituents possess useful properties concerning human health. Many of them may be applied in anticancer therapy, cardiovascular and nervous system disorders to reduce the level of cholesterol, to regulate the glucose level or to stimulate hormone production [22].

\section{Conclusion}

Essential oil of rosemary showed a remarkable potency against four of the most notorious bacteria and exhibited allelopathic impact against Chlorella vulgaris, which is causing a contaminant factor for our cyanobacterial isolates. Phytochemical components of Rosemary essential oil had been screened and isolated in many researches and other studies could be conducted in order to test antagonistic activities for each component in order to recommend firms of pharmacy to manufacture these compounds and applied as alternative medicinal therapy against the broad-spectrum drug resistant pathogens. 


\section{References}

[1]. K.R. Narayana, M.S. Reddy, M.R. Chaluvadi and D.R.Krishna. Bioflavonoid classification, pharmacological, Biochemical effects and therapeutic potent. Indian J pharmol, 33, 2001, 2-16.

[2]. S.M. Shah, F.A. Khan, S.M.H. Shah, K.A. Chishti, S.M. Pirzada, M.A. Khan and A. Farid. Evaluation of phytochemicals and antimicrobial activity of white and blue capitulum and whole plant of Silybum marianum. World Appl Sci J, 12, $2011,1139-1144$.

[3]. S. Demissew, E. Dange Basic and Applied Research on Medicinal Ethiopia, In: Proceedings of national workshop on conversation and sustainable Use of Medicinal plants in Ethiopia, Addis Ababa, 2001, 29.

[4]. S. Prabuseenivasan, M. Jayakumar and S. Ignacimuthu In vitro antibacterial activity of some plant essential oils. BMC Complement Altern Med, 6, 2006, 1-8.

[5]. A. Hussain, F. Anwar, A. Chatha, A. Jabbar, S. Mahboob, and P. Nigam, Rosmarinus officinalis essential oil: antiproliferative, antioxidant and antibacterial activities. Brazilian Journal of Microbiology, 41, 2010, 1070-1078.

[6]. M. Minaiyan, AR. Ghannadi, M Afsharipour, P. Mahzouni .Effects of extract and essential oil of Rosmarinus officinalis L. on TNBS-induced colitis in rats. Res Pharm Sci, 6, 2011, 13-21.

[7]. A. M. Najem, I. J. Abed and A. M. al Haidarry. Evaluation the anti Cyanobacterial effect of Rosemary (Rosmarinus officinalis L.) Essential oil. Iraqi Journal of Biotechnology, 15, 2015, 1, 97-102.

[8]. K.E. Apt and P.W. Brehrens. Commercial developments in micro algae biotechnology.Journal of Phycology, 35, 1999, $215-226$.

[9]. Jamshidi, R., Afzali, Z. and Afzali, D. Chemical composition of hydrodistillation essential oil of rosemary in different origins in Iran and comparison with other countries. American-Eurasian J. Agric. \& Environ. Sci. 5, 2009, 1, 78-81.

[10]. I. J. Abed, A. A. Al-Hussieny, R. F. Kamal and A. M. Jawad. Environmental and identification study of algae present in three drinking water plants located on Tigris River in Baghdad. International Journal of Advanced Research, 2, 2013, 3, 895-900.

[11]. D. Kim, T. Oda, T. Muramatsu, Kim, Daeil, Y. Matsuyama, and T. Henjo. Possible factors responsible for the toxicity of Cochlodinium polykrikoides, a red tide phytoplankton. Comparative Biochemistry and Physiology, 132, 2002, 415-423.

[12]. Clinical and Laboratory Standards Institute (CLSI). Microdilution method. Methods and dilution antimicrobial susuptibility test for bacteria that grow aerobically. 32, 2012, 2. Ninth ed. USA, 16-18.

[13]. A. Budzynska, M. Wieckowska-Szakiel, B. Sadowska, D. Kalemba, and Rozalska, B. Antibiofilm activity of selected essential oils and their major components. Polish J. of Microbiology. 60(1), 2011, 35-41.

[14]. H. Wang, F. Liang and L. Zhang. Composition and Anti-cyanobacterial Activity of Essential Oils from Six Submerged Macrophytes. Pol. J. Environ. Study, 24, 2015, 1, 333-338.

[15]. G. A. Abdulhasan. The Biological Effect of Rosmarinus officinelis L. Essential Oil on Biofilm Formation and Some Fimbrial Genes (fimH-1 and mrkD) of Klebseilla pneumonia. Iraqi Journal of Science. Vol 56, 2015, 3, 2553-2560.

[16]. M. Sienkiewicz, M. Łysakowska M. Pastuszka W. Bienias and E. Kowalczyk. The Potential of use basil and Rosemary Essential Oils as Effective Antibacterial Agents. Molecules, 18, 2013, 9334-9351.

[17]. G. G. F. Nascimento, L. Juliana P. C. Freitas and G. L. Silva. Antibacterial activity of plant extracts and phytochemicals on antibioticresistant bacteria. Brazilian Journal of Microbiology, 31, 2000, 247-256.

[18]. P.Lopez, C. Sanchez, R. Battle and C. Nerin. Solid- and vapor-phase antimicrobial activities of six essential oils: Susceptibility of selected foodborne bacterial and fungal strains. Agric. Food Chem. 53, 2005, 6939-6946.

[19]. S. Prabuseenivasan, M.Jayakumar and S. Ignacimuthu. . In vitro antibacterial activity of some plant essential oils. BMC Complement. Altern. Med., 6, 2006, 39.

[20]. A. Fabio, C. Cermelli, G. Fabio; P. Nicoletti and P. Quaglio. Screening of the antibacterial effects of a variety of essential oils on microorganisms responsible for respiratory infections. Phytother. Res., 21, 2007, 374-377.

[21]. M.S. Bendeddouche, H. Benhassaini, Z. Hazem and A. Romane. Essential oil analysis and antibacterial activity of Rosmarinus tournefortii from Algeria. Nat. Prod. Commun, 6, 2011, 1511-1514.

[22]. A.E. Edris. Pharmaceutical and therapeutic potentials of essential oils and their individual volatile constituents: A review. Phytother. Res., 21, 2007. 308-323. 